\title{
Gestión de Empresas 2.0. Desde la Estructura Jerárquica hasta las Redes de Conocimiento. El Modelo CIACO_RED
}

\author{
Business Management 2.0. Since the hierarchical structure to Knowledge \\ Networks. Model CIACO_RED
}

\author{
Lourdes Sáiz, Miguel Ángel Manzanedo, Ricardo del Olmo y Roberto Alcalde \\ Dpto. de Ingeniería Civil. Escuela Politécnica Superior. Universidad de Burgos. C/ Francisco de Vitoria, s/n, 09006 Burgos. \\ Isaiz@ubu.es,mmanz@ubu.es,rdelolmo@ubu.es, radelgado@ubu.es
}

Fecha de recepción: 7-9-2011

Fecha de aceptación: 19-9-2011

\begin{abstract}
Resumen: El objetivo de este trabajo es diseñar un modelo de gestión que permita hacer frente a tres de los principales fenómenos que afectan a la empresa actual: la explosión científico-tecnológica, la globalización de los mercados y la existencia de revolucionarias tecnologías de comunicación, colaboración e integración, en particular el desarrollo de Internet. El resultado es el modelo CIACO_RED, cuyos componentes son: las capacidades necesarias para detectar las oportunidades y amenazas de la empresa; la identificación de los requerimientos de los nuevos clientes a los que atender, el aprendizaje colectivo permanente y las redes de conocimientos virtuales y globales.
\end{abstract}

Palabras clave: gestión de empresas, redes de conocimiento, globalización, avances científico-tecnológicos, tecnologías 2.0. modelo CIACO_RED

\begin{abstract}
The aim of this work is to design a management model that can cope with three major events affecting the company today: the scientific and technological explosion, globalization of markets and the existence of revolutionary technologies for communication, collaboration and integration, in particular the development of the Internet. The result is CIACO_RED model, whose components are: the skills to identify opportunities and threats of the company, identifying the requirements of the new customers we serve, ongoing collective learning and knowledge networks and global virtual.
\end{abstract}

Keywords: business management, knowledge networks, globalization, scientific and technological advances, technologies 2.0. CIACO_RED model.

\section{Presentación, alcance y objetivo del estudio}

En este trabajo se abordan tres de los principales fenómenos que convierten a la empresa en una estructura en constante cambio y donde las personas participativas, colaborativas, abiertas, con visión global, y sin miedo a lo nuevo e insólito, se convierten en los protagonistas principales y agentes capaces de dar respuestas a los nuevos desafíos. El resultado es una nueva organización, donde pueden participar agentes desconocidos hasta el momento, que deberá aprender a reinventarse de forma permanente y en clara sintonía con tales cambios. A este respecto, no es mucho decir, que el éxito de la empresa, su fuerza, energía y permanencia no está en sus fuentes de producción, sino en el conocimiento, talento y competencias de sus trabajadores, actuando colectivamente, adaptados a las nuevas tecnologías y sin miedo a lo nuevo e inesperado.

Obviamente, ante esta coyuntura, los modelos o formas de gestión tradicionales no sirven y deben ser sustituidos por otros que presten su atención de manera prioritaria en los lazos que articulan la empresa y que, además, son los facilitadores del uso pleno de las capacidades y conocimientos de los trabajadores. Este planteamiento organizativo, en el pasado, sencillamente, no era posible.

Se habla, así, de empresas 2.0 (McAfee, 2006), modelos de gestión abiertos, redes de conocimiento como alternativas de interacción y colaboración para acometer los nuevos desafíos y todo un conjunto de 
tecnologías para la información, relaciones y comunicaciones al servicio de los «trabajadores del conocimiento» (Davenport y Prusak, 1998; Drucker, 1999), porque, en efecto, hoy, los agentes de la empresa o trabajan con el conocimiento o no trabajan con nada. Nos interesa, igualmente, el papel que debe desempeñar la dirección, pues, estos nuevos requisitos, formas emergentes, categorías de estos agentes, producción de conocimientos y su necesidad de intercambiarlos y compartirlos, tecnologías novedosas, potentes $y$ atractivas, etc., sin embargo, no aparecen y se usan espontáneamente, sino que dependen, en gran medida, de la estrategia formulada, las decisiones que se adopten y las acciones que se acometan por los directivos de la empresa.

Así pues, el presente artículo comienza con unas reflexiones acerca de los fenómenos más significativos que alertan de escenarios empresariales desconocidos, pero con inmensas oportunidades para la empresa y reglas de juego totalmente distintas. Después se hace una incursión a las redes de conocimiento, como elementos para crear espacios de interrelación y conocimientos, capaces de afrontar los desafíos actuales y solucionar los difíciles problemas de la empresa y seguidamente se plantea una aproximación a la gestión de esta nueva empresa, diseñando un modelo propio, el modelo CIACO_RED, que integra las capacidades necesarias para conocer las oportunidades y amenazas; la identificación de los requerimientos de los nuevos clientes a los que atender, el aprendizaje colectivo permanente y las propias redes de conocimiento.

Este planteamiento de gestión, para tener éxito, necesita estar arropado y sustentado por las tecnologías Web 2.0, sin ellas sería imposible y además, contrariamente a lo que se piensa, la gestión de estas nuevas realidades empresariales no es tener la certeza de lo que va a pasar, sino contar con las habilidades para navegar por ese océano infinito de incertidumbres y posibilidades sin precedente hasta ahora. El apartado de conclusiones y bibliografía completan el presente trabajo.

\section{Un nuevo contexto para la actuación y desarrollo de la empresa. Algunas reflexiones}

La evolución y velocidad sin precedentes de los cambios del entorno, en el que transcurre la actividad de las empresas, presentan nuevos escenarios y desafíos a los que hacer frente, puesto que una falta de capacidad de respuesta a tiempo puede llevar a la empresa a dejarla fuera de juego. Estos cambios se pueden resumir en tres importantes fenómenos: la explosión científico-tecnológica, la globalización de los mercados y la existencia de revolucionarias tecnologías de comunicación, colaboración e integración.

La globalización representa que cualquier empresa puede generar negocios en cualquier lugar, con quién desee y durante el tiempo que quiera. La contrapartida es que, de igual manera, puede tener competidores a lo largo y ancho de todo el planeta, provocando que su oferta se quede obsoleta con inusitada rapidez. La consecuencia inmediata, a efectos del trabajo que se expone en este artículo, es una expansión de los mercados como no se había producido antes, al igual que una intensificación de la competencia, lo que permite imaginar la creación de infinitos nichos de actividad para las empresas, actualizar y renovar los existentes, así como el descubrimiento de negocios totalmente nuevos.

No olvidamos, sin embargo, que todo ello va unido a brotes más o menos intensos de inestabilidad e incertidumbre que, lejos de considerarlos fenómenos de carácter negativo, una vez superados, contribuyen a alimentar y hacer crecer, si cabe más, esta expansión de mercados, sectores, negocios y productos. En todo caso, la presión de los avances permanentes en la ciencia y la tecnología, así como el aumento imparable de la globalización deviene, para la empresa, en una obsesión por adquirir la agilidad necesaria para poder competir con garantías de éxito y ganar la dinámica de los negocios, con ciclos de vida de los productos cada vez más cortos y atención a los clientes cada vez más personalizada.

El tercer elemento de cambio en el mundo y los negocios está relacionado con el desarrollo de las comunicaciones y en particular con Internet. Para ello nos remitimos, teniendo en cuenta igualmente las críticas y opiniones de otros autores (Ghemawat, 2007; OXFAM, 2007) a los postulados de Friedman (2006) que apunta diez «aplanadores» para conseguir reducir las diferencias entre países desarrollados y emergentes, entre empresas grandes y pequeñas, o entre profesionales dentro y fuera de las empresas, de modo que los agentes, independientemente de su tamaño, localización u oferta, pueden participar de negocios a escala mundial (para la empresa actual es más importante tener una buena página web que un edificio lujoso). Estos aplanadores se agrupan en tres categorías: a) plataforma de comunicación global (internet, workflow software, etc.), b) formas novedosas de colaboración (outsourcing, offshoring, 
opensourcing, etc.) y c) esteroides que potencian las capacidades de otros aplanadores (virtualización, digitalización, personalización, etc.). Llegamos, así, a uno de los puntos más relevantes para este trabajo, que es la adquisición y generación, por parte de las personas, de capacidades inimaginables hasta hace pocos años.

Es decir, el agente de transformación central y el que puede dar respuesta a esta vorágine es, sin duda alguna, el individuo y su capacidad de constituir redes y organizarse en ellas. Con las nuevas tecnologías colaborativas esto es posible (Margalina, 20 I0). Más concretamente, el epicentro de este fenómeno son las redes abiertas, colaborativas y globales entre individuos, empresas, negocios, etc. donde tan sólo se requiere un acceso a internet para participar competitivamente; es la llamada Web 2.0 (O'Reilly, 2005).

Desde el punto de vista organizativo, estas plataformas han facilitado llevar a cabo procesos de comunicación y colaboración de los conocimientos a escala empresarial (y también global, en el sentido más amplio del uso de las redes) y han posibilitado que especialistas de distintos departamentos (o del mismo, si es el caso), con diversos niveles de saber y experiencia, utilicen y actualicen sus conocimientos, se coordinen y los orienten hacia la práctica y los problemas emergentes de forma rápida y dinámica. De hecho, la revolución y avances inusitados de bastantes sectores, como el de las telecomunicaciones, el aeroespacial, la informática, la sanidad o la formación, por ejemplo, dan prueba de ello. No olvidamos, además, la superioridad competitiva de los grupos interrelacionados sobre aquellos que piensan y actúan de manera individual. Las redes de conocimiento son la expresión más cercana a estas interrelaciones.

\section{Las redes de conocimiento en la empresa}

Para acercarnos a la redes de conocimiento en el ámbito empresarial se van a tratar algunas cuestiones relacionadas con su finalidad, los valores asociados para que sea posible su existencia y permanencia, ciertas características reveladoras de las mismas o los elementos que favorecen -o inhiben- su desarrollo. Para ello, además de analizar los trabajos e investigaciones de varios autores, también se han estudiado y discutido dos iniciativas de gran interés, que se constituyen en sendos proyectos, desarrollados gracias a los Programas Marco de Investigación y Desarrollo en Tecnologías de la Información de la Unión Europea. Son el ECOLEAD, en http://ecolead.vtt.fi, y el COIN, en www.coin-ip.eu. Estos últimos para los aspectos más concretos, vinculados con el valor agregado que generan los negocios colaborativos, las cuestiones de diseño e implantación de organizaciones basadas en redes de colaboración y los aspectos de gestión, información y difusión permanentes de este tipo de comunidades.

Empezamos acercándonos a la definición o concepto de las redes de conocimiento y aunque hay muchas y variadas, propuestas por otros tantos autores (Casas, 200 I; Artiles, 2002; Faloh, 2002; Yoguel y Fuchs, 2003; Álvarez Merino, 2004), nos interesa resaltar que las redes de conocimiento, ya sean entre agentes internos, externos o de ambos, es un grupo, en general, multidisciplinar de personas que se asocian de manera intencionada no sólo para desarrollar, mejorar y aumentar la eficacia y calidad del trabajo, sino también, para crear y fortalecer la cooperación, aprovechar los recursos y capacidades disponibles y posibilitar el libre flujo de conocimiento entre sus miembros. Todo ello, con el uso intensivo de las adecuadas y actualizadas tecnologías de la información y las comunicaciones.

Lo anterior exige que la actividad de los partícipes se fundamente en principios como la solidaridad, cooperación e integración; es decir, al mismo tiempo que se acomete, e incluso se acepta ya con cierta naturalidad, una globalización de los mercados o del conocimiento, se está requiriendo, en el marco de las redes de conocimiento, una globalización de la solidaridad, confianza y cooperación. Otra manera interesante para reconocer las redes de conocimiento es considerarlas como un mecanismo, preferentemente, de relaciones -más virtuales que presenciales- entre los agentes para promover el intercambio y compartición del conocimiento, utilizar o desarrollar prácticas de trabajo exitosas, colaborar en iniciativas de capacitación, creatividad e investigación y aumentar los conocimientos por medio de la reciprocidad, confianza e intercambio.

Según recoge Álvarez Merino (2004), una red de conocimiento se justifica cuando se identifica un conocimiento acumulado disponible (...), y en cuanto existe y puede ser generado, se debe dirigir la demanda para el uso de tal conocimiento en productos o servicios, a fin de mantener y consolidar los mercados internos y externos. De manera sintética, y en palabras de Lopera (2000), toda red de conocimiento, para que tenga éxito, exige compromiso con la práctica participativa, una estructura de manejo, normas claramente definidas, objetivos y reglas, implicación de los miembros, recursos humanos y financieros y 
un enfoque de aprovechamiento de las nuevas tecnologías, que son las que pueden mejorar y fortalecer el proceso social al que aspira la red. Este último aspecto se relaciona con una plataforma metodológica para la comunicación y la gestión del conocimiento, que no se puede abordar con estructuras jerárquicas.

En el ámbito de la empresa, aunque las redes de conocimiento proporcionan múltiples e interesantes beneficios, aquí se quiere incidir en su contribución a la construcción de capital social (Thompson, 2008), dado que los intercambios basados en la confianza, solidaridad y reciprocidad aceleran y mejoran el aprendizaje de todos los miembros y consiguen una ganancia neta de conocimiento dinámico, que es, sin duda, el principal ingrediente para conseguir las ventajas competitivas. En efecto, como señala Giuliani (2002), uno de los elementos que caracteriza, distingue y determina el éxito o fracaso de la red es su capacidad para absorber el conocimiento y las capacidades, es decir, para identificar, asimilar y explotar conocimientos, provenientes de fuentes internas y externas a ella.

Merece la pena destacar, para su reflexión, que, como apuntan Pérez y Castañeda (2009), las redes de conocimiento generalmente se desarrollan en el marco de tres elementos reveladores, que son: I) la existencia de fuertes incertidumbres estratégicas, 2) avances técnicos que todavía no se han apropiado y estabilizado de manera generalizada y 3) algún grado de libertad del patrón tecnológico predominante (éste último citado por Malerba et al., 1999).

Por su parte, la estructura/arquitectura de la red o forma en que se agrupan sus miembros puede ser muy variada. Aquí apuntamos una propuesta (adaptada de Pérez y Castañeda, 2009) basada en «clusters», cada uno de los cuales se identifica o responsabiliza de diversos ámbitos o temas orientados hacia unos objetivos comunes, que pueden, a su vez, conformarse por unidades más básicas, denominadas grupos de conocimiento. Estos grupos constituyen el recurso humano esencial, por lo que es imprescindible garantizar el éxito de sus procesos y la consecución de los objetivos planificados para cada grupo.

La arquitectura se completa con uno o varios «centros» de red, cuyo cometido es garantizar el aprovechamiento y difusión de los conocimientos adquiridos en cada cluster, coordinarse entre sí y con otros centros para el logro de los objetivos de la red y de las actividades encomendadas. Todos los componentes de la red interactúan entre sí y el conoci- miento, las capacidades, las buenas prácticas, los avances en los métodos o técnicas, se van integrando, compartiendo y utilizando en la red de conocimiento.

Las redes de conocimiento deben disponer, además, de un espacio virtual donde estén a su disposición no solo las herramientas de información y comunicación más actuales y los servicios de conocimiento interactivos que se necesitan, sino lo más importante, posibilitar de manera natural la adquisición o consulta del conjunto de conocimientos, capacidades, métodos, etc., además de facilitar la generación de otros nuevos.

Sin duda, la implantación y desarrollo pleno de las redes de conocimiento no está exento de dificultades y restricciones, vinculadas no tanto con disponer de las tecnologías adecuadas, sino, principalmente, con la difusión y canalización del conocimiento y el acceso a los métodos formales e informales de aprendizaje, que generan grupos desiguales o brechas de conocimiento, problemas de derechos de propiedad, dificultades en el aseguramiento de la calidad del conocimiento existente en la red, así como los dilemas que plantea el almacenamiento y codificación del conocimiento.

\section{La gestión de la nueva empresa. EI modelo CIACO_RED}

Descritos sucintamente los fenómenos con los que tratan las empresas en la actualidad, ahora es el momento de plantear un marco o esquema de gestión que dé respuesta a estas nuevas realidades, cuyos componentes principales son la identificación, creación y apropiación de prácticas de gestión efectivas para este nuevo entorno y el desarrollo de habilidades para implantar tales prácticas de manera efectiva. En definitiva, estamos hablando de una Gestión de Empresas 2.0, donde se destaca la colaboración entre los agentes de conocimiento, como rasgo más distintivo, y se corresponde con la generación de espacios propios proclives y favorecedores de lo nuevo, lo inesperado y lo insólito, que sean válidos para los clientes. Las respuestas a estos escenarios vienen de la mano de tecnologías significativas capaces de establecer lazos que unan y articulen la empresa y faciliten el trabajo del conocimiento de una forma que en el pasado no era posible.

En esta misma línea, Hamel (2008) apunta que las empresas disponen de procesos del siglo XXI, facilitados por Internet, mientras que los correspondientes a la 
gestión son de mediados del XX, pero, incluso, basados en los que sirvieron para la gestión de las empresas en el siglo XIX.Vázquez (2008), va más allá afirmando que los estilos y procedimientos de gestión utilizados hasta el momento, han sido incapaces de interpretar, y mucho menos, diagnosticar la influencia para la empresa de los nuevos fenómenos emergentes. Se apunta, pues, a un cambio de paradigma, porque las bases más importantes de la empresa se comportan de forma distinta, entre éstas, las ideas, el conocimiento, los valores, la responsabilidad, las personas, los grupos, la estructura organizativa, las tareas, la toma de decisiones, la asignación de recursos, los clientes, los productos y servicios, etc.

Nuestra contribución a este cambio de paradigma, nos lleva a plantear un modelo de gestión de empresas, denominado CIACO_RED (figura I), que creemos puede servir para acelerar el ritmo de renovación estratégica en la empresa, de manera constante y permanente, sin necesidad de crisis o traumas, muy difíciles de superar. Sin duda, la base para ello reside en la innovación, es más, sin ésta es imposible avanzar y conseguir resultados en la gestión de las nuevas realidades. El modelo CIACO_RED se construye integrando los siguientes componentes:

I. Conjunto de capacidades para detectar las nuevas oportunidades y amenazas, que permitan saber moverse en el mundo.

2. Diseño de identidades valiosas para las nuevas generaciones de clientes.

3. Desarrollo de un aprendizaje colectivo permanente.

4. Establecimiento de redes de conocimiento virtuales y globales.

Figura I

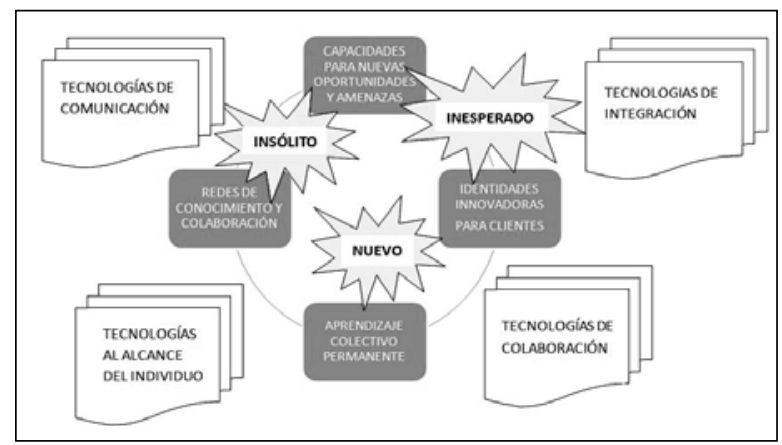

Modelo CIACO_RED de Gestión de Empresas 2.0

El primer componente del modelo, capacidades que sirvan para detectar amenazas y oportunidades, se basa, necesariamente, en la creatividad y el intento decidido, por parte de la empresa y trabajadores, de potenciarla al máximo, creando entornos atractivos en los que la creatividad sea una realidad. En ocasiones, los propios sistemas de gestión, la rutina, la falta de responsabilidad, el miedo al error, la aversión al riesgo, etc. son una rémora para la creatividad. Las organizaciones que, a la larga, son más innovadores y consiguen los niveles de éxito previstos son las que permiten a sus trabajadores equivocarse, a los clientes devolver el producto o reclamar un servicio y a los proveedores suministrar una mercancía con algún defecto. Y esto es así, porque lo importante es aprender de estas situaciones, superarlas y mejorarlas permanentemente.

Precisamente, la necesidad de una mayor creatividad implica cambiar la manera de gestionar, porque se requieren sistemas, procedimientos y funciones que intencionadamente ayuden y aprovechen las capacidades de los trabajadores todos los días y en todos los momentos, primando el conocimiento, compromiso, responsabilidad y, sobre todo, la visión, misión y objetivos compartidos, con un liderazgo que cree valor (Goleman et al., 2009). Pasamos así, de la estructura jerárquica y la obediencia del trabajador, o casi, a la responsabilidad individual, compromiso y toma de decisiones participativa en función del conocimiento y las redes de colaboración del individuo o los grupos. Son empresas donde todos participan activamente, generando valor económico y social.

El segundo elemento se centra en dar respuesta a las necesidades de una nueva generación de clientes, cuyo rasgo más distintivo es que son personas conectadas entre sí. De modo que cuanto más interactúen entre ellos, más dependerá la empresa, no sólo de la creatividad de sus trabajadores, comentada en las líneas anteriores, sino también de las nuevas tecnologías para posicionar correctamente los productos y no quedar olvidada. Otro aspecto muy destacado son las relaciones de confianza que se han de establecer con los clientes y viceversa, para participar, crear, aprender y dar soluciones, sin temor a prejuicios, ni complejos pueriles.

Así es como las fronteras de la empresa se están volviendo invisibles, líquidas, transparentes, como lo es la información que tienen los clientes, caracterizada por ser libre, ubicua, accesible, cercana, fácil. Con estas nuevas realidades, hay que pasar de una gestión sustentada en el control y las jerarquías a otra diametralmente distinta concebida para diseñar, fomentar y estimular las redes abiertas, donde se cul- 
tiva la confianza y se cuida a los clientes, participantes y sus intereses. Igualmente, de una gestión basada en productos estandarizados, o casi, al diseño y gustos a medida de los nichos de clientes (Anderson, 2009), e incluso pensados y diseñados por ellos mismos, como culmen de innovación y creatividad.

Los dos elementos anteriores derivan en un aprendizaje colectivo permanente que se inicia como un proceso reflexivo individual hasta llegar a una comprensión social. Es decir, las empresas no aprenden en abstracto, sino por medio de sus miembros y de la capacidad de éstos para integrar, comunicar, compartir y comprometerse con el conocimiento que cada uno, por separado, posee. Así pues, para que este proceso colectivo permanente tenga éxito es imprescindible que los miembros de la empresa sepan y practiquen el «aprender a aprender»».Y en este aprender constantemente a lo largo de la vida, de nuevo las soluciones tecnológicas, como el e-learning, permiten, salvo excepciones, disponer permanentemente de capacitación y facilitar la actualización de contenidos, que con las metodologías tradicionales de formación puede ser imposible.

Se pasa así de una gestión aislada y centrada en el trabajo individual e independiente a otra donde se fomenta la confianza entre los participantes, se valora la colaboración y se premia a los miembros por intercambiar, compartir y valorar el conocimiento colectivo.

El último elemento, como colofón del modelo propuesto, se refiere a las redes de conocimiento, como expresión intencionada de que el conocimiento es la base de cualquier tipo de red y por ello, las redes humanas, en esencia, son redes de conocimiento (Faloh, 2002). Teniendo en cuenta lo comentado en el apartado 3, interesa, aquí, reflexionar sobre el nivel de conocimientos, competencias y destrezas de las personas que trabajan en grupo, frente a las que actúan de manera individual. Se pone de manifiesto con bastante nitidez que se trata de mecanismos sobre todo de intercambio social que relacionan a diferentes miembros de la empresa, para promover el intercambio de conocimiento, compartir prácticas y metodologías, colaborar en nuevas iniciativas y, lo que es más importante, aumentar el conocimiento por las complementariedades, la reciprocidad y la confianza.

Descritos los componentes sobre los que se construye este nuevo modelo de gestión, sólo resta apuntar que es necesarios establecer las condiciones más adecuadas para la implantación y desarrollo de esta gestión innovadora, que consiste en no reproducir el éxito del pasado, sino en generar espacios proclives a la novedad y la sorpresa, junto con disponer de las nuevas tecnologías y usarlas, igualmente, con mentalidad colaborativa y global. De ello nos ocupamos en el siguiente apartado.

\section{Los Dominios de la Dirección de Empresas 2.0}

La importancia de la dirección se justifica en la habilidad de implantar el modelo de gestión diseñado, siendo necesario, al menos, cuatro ámbitos fundamentales de actuación, que coinciden con los elementos propuestos, la visión del entorno, los requerimientos de valor para el cliente, el aprendizaje organizativo permanente y la gestión de las redes de conocimiento. Pero hay más, cuando la organización se enfrenta a desafíos que requieren soluciones desconocidas, como es el caso, esto siempre va unido a una cultura organizativa receptiva, cuyo propósito es lograr una comunidad sólida y obtener la confianza de los trabajadores.

La visión del entorno se relaciona con potenciar, estimular y premiar las capacidades para detectar las oportunidades y acometerlas de forma decidida y a tiempo, así como su transformación en productos o servicios válidos para el cliente, que la empresa deberá diseñar, construir y distribuir de manera rápida.

Los requerimientos de valor para el cliente enlaza con el domino anterior y se trata de plasmarlo en oferta que satisfaga al cliente y en la búsqueda permanente de innovaciones generadoras de valor para todos los participantes. Aquí, los directivos deben conocer muy bien a estas nuevas generaciones de clientes — sus necesidades, expectativas y deseos- y reinventar de forma permanente los productos, servicios e identidades dirigidos a ellos.

La producción, desarrollo y mantenimiento de aprendizaje colectivo permanente es vital para el éxito del proyecto empresarial. Todas las capacidades y conocimientos desplegados han de mantenerse actualizados constantemente y de manera coordinada. Aquí está el futuro y las posibilidades de la empresa, en el aprendizaje y la comunicación, y los directivos deben actuar a modo de maestros, diseñando los objetivos del aprendizaje, la manera de lograrlos y los beneficios de su aplicación.

Sin embargo, como señalan Camarinha-Matos y Afsarmanesh (2004), la gestión de las redes de conoci- 
miento y compromiso no es automática, se debe atender explícitamente. Además, la importancia y consecuencias de las redes radica en que precisamente, la socialización del conocimiento le permite a la empresa percibir más fácilmente los nuevos escenarios y, en consecuencia, tomar decisiones más seguras en cada oportunidad.También, las plataformas tecnológicas propician colaboraciones que probablemente no habrían ocurrido en otro caso, mientras que la incomunicación, falta de colaboración o ausencia de integración no tiene sentido ante los desafíos de la nueva empresa.

Estos cuatro dominios significan la ineludible labor que han de desplegar los nuevos directivos y lo que es más importante, la necesidad de su comprensión efectiva para el éxito de la nueva gestión empresarial, sin la cual no es posible la empresa 2.0.

\section{Discusión y Conclusiones}

Las conclusiones más relevantes del presente trabajo son, que la globalización de los mercados, los avances en la ciencia y la tecnología y los extraordinarios desarrollos de las tecnologías de la comunicación y colaboración, lejos de representan peligros, ofrecen a la empresa enormes oportunidades de negocio. Para ello, se requiere una nueva empresa que comprenda las incertidumbres y los cambios y los sepa gestionar. Con base en la colaboración entre los agentes de conocimiento y desarrollando modelos de gestión, como el CIACO_RED planteado en este trabajo, es posible dar respuestas adecuadas a estos desconocidos escenarios.

Esta novedosa gestión, que integra muy destacadamente las redes de conocimiento, las relaciones de confianza, colaboración y compromiso y el papel activo de la dirección, permite determinar un conjunto de elementos, tales como las capacidades necesarias para competir en globalización; el conocimiento de las nuevas generaciones de clientes, con sus necesidades y expectativas; la importancia de trabajar en red o el apoyo decidido al aprendizaje organizativo permanente, para enfrentar los nuevos y apasionantes desafíos que tiene en estos momentos la empresa actual. Los modelos de gestión tradicionales no pueden sustentar el «nuevo edificio virtual» que se ha proyectado para dar cobijo a los negocios actuales $y$ futuros.

\section{Bibliografía}

ANDERSON, CH. (2009), La Economía Long Tail. Empresa Activa. Barcelona.
ÁLVAREZ MERINO, J. (2004), Gestión del Conocimiento y Desarrollo Regional, en http://www.gestiopolis.com/recursos/documentos/fulldocs/gerl/gescondesreg.html/.

ARTILES, S. (2002), Las Redes de Conocimiento como Producto de la Gerencia de Información en Ambientes Académicos, en Faloh, R. y Fernández, M.: Gestión del Conocimiento: Concepto, Aplicaciones y Experiencias. Empresa de la Gestión del Conocimiento y la Tecnología. La Habana.

CAMARINHA-MATOS, L.; AFSARMANESH, H. (2004) (eds). Collaborative Networked Organizations: A Research Agenda for Emerging. Springer. USA.

CASAS, R. (200 I), La Formación de Redes de Conocimiento. Una Perspectiva Regional desde México. Instituto de Investigaciones Sociales (UNAM). México.

DAVENPORT,T.; PRUSAK, L. (1998), Working Knowledge: How Organizations Manage What They Know. Harvard Business School Press. Boston, Massachusetts.

DRUCKER, P. (1999), «Knowledge-Worker Productivity». Management Review,Vol. 4I, No. 2, pp. 79-94.

FALOH, R. (2002), Redes del Conocimiento. Empresas de Clase en Cuba, en Faloh, R.y Fernández, M.: Gestión del Conocimiento: Concepto, Aplicaciones y Experiencias. Empresa de la Gestión del Conocimiento y la Tecnología. La Habana.

FRIEDMAN,T. (2006), The World is Flat. Douglas \& Mclntyre Ltd. Canadá.

GHEMAWAT, P. (2007), «Why the World Isn't Flat?» In Foreign Policy 159, The Economist: 18/0 I/07.

GOLEMAN, D.; KAUFMAN, P.; RAY, M. (2009), El Espíritu Creativo. Zeta. Barcelona.

GULIANI, E. (2002), Cluster Absorptive Capability: An Evolutionary Approach for Industrial Clusters in Developing Countries. Proceeding Conference on «Industrial Dynamics of the New and Old Economy -Who is Embracing Whom?».

HAMEL, G. (2008), «The Facebook Generation vs the Fortune 500», en http://blogs.wsj.com/management/2009/03/24/the-facebook-generation-vs-the-fortune-500/.

LOPERA, H. (2000), Integración de Redes de Conocimiento, en http://www.udea.edu.co/hlopera/irc.html.

MALERBA, F. ET AL. (1999), «History Friendly Models of Industry Evolution:The Computer Industry». Industrial and Corporate Change, Vol. 8, No. 2, pp. 3-40.

MARGALINA, V. (20I0), La web 2.0 y la co-creación de valor. Impacto de la web 2.0 en la empresa y el aprovechamiento de la inteligencia colectiva. Tesis Doctoral. Universidad Rey Juan Carlos. 
MCAFEE, A. (2006), «Enterprise 2.0: The Dawn of Emergent Collaboration». MITSloan Management Review, Vol. 47, No. 3, pp. 20-28.

OXFAM INTERNATIONAL (2007), Signing Away the Future. Oxfam Briefing Paper, March.

O'REILLY,T. (2005): «What is Web 2.0» en http://oreilly.com.

PÉREZ, Y.; CASTAÑEDA, M. (2009): «Redes de Conocimiento». Ciencias de la Información,Vol. 40, No. I, pp. 320.
THOMPSON, K. (2008), The Networked Enterprise: Competing for the Future Through Virtual Entreprise Networks. Meghan-Kiffer Press.

VÁZQUEZ,A. (2008), Estrategias de la Imaginación: Innovación y Conocimiento en las Sociedades de Control. Granica, Barcelona.

YOGUEL, G.; FUCHS, M. (2003), Desarrollo de Redes de Conocimiento. Estudios sobre Empleo, en http:// www.mecon.gov.ar/crecimiento/5_estudios/2_empleo/d_desarrollo_redes_conocimiento.pdf. 